\title{
New Ansatz for Metric Operator Calculation in Pseudo-Hermitian Field Theory
}

\author{
Abouzeid M. Shalaby* \\ Physics Department, Faculty of Science, Mansoura University, Egypt.
}

\begin{abstract}
In this work, a new ansatz is introduced to make the calculations of the metric operator in Pseudo-Hermitian field theory simpler. The idea is to assume that the metric operator is not only a functional of the field operators $\phi$ and its conjugate field $\pi$ but also on the field gradient $\nabla \phi$. Rather than the locality of the metric operator obtained, the ansatz enables one to calculate the metric operator just once for all dimensions of the space-time. We calculated the metric operator of the $i \phi^{3}$ scalar field theory up to first order in the coupling. The higher orders can be conjectured from their corresponding operators in the quantum mechanical case available in the literature. We assert that, the calculations existing in literature for the metric operator in field theory are cumbersome and are done case by case concerning the dimension of space-time in which the theory is investigated. Moreover, while the resulted metric operator in this work is local, the existing calculations for the metric operator leads to a non-local one. Indeed, we expect that the new results introduced in this work will greatly lead to the progress of the studies in Pseudo-Hermitian field theories where there exist a lack of such kind of studies in the literature. In fact, with the aid of this work a rigorous study of a $\mathcal{P} \mathcal{T}$-symmetric Higgs mechanism can be reached.
\end{abstract}

PACS numbers: 12.90.+b, 11.30.Er, 12.60.Cn

Keywords: non-Hermitian models, $\mathcal{P} \mathcal{T}$-symmetric theories, pseudo-Hermitian theories, metric operator.

*Electronic address: amshalab@ mans.edu.eg 
The subject of quantum mechanical pseudo-Hermitian models has attained extensive attention in the literature [1, 2, 3, 4, 5, 6, 7, 8, 9, 10, 11, 12, 13, 14, 15, 16, 17]. Two main problems have appeared at the beginning of these studies, namely, the indefinite norm and unitarity problems. In fact, there exists two equivalent approaches to solve these problems. The first approach, due to Bender et. al, is to replace the bra in the Dirac convention by a CPT bra such that the new inner product takes the form [18]

$$
\langle A \mid B\rangle_{C P T}=(C P T|A\rangle)^{T}|B\rangle
$$

which replaces the conventional Dirac bracket $\langle A \mid B\rangle=|A\rangle^{\dagger}|B\rangle$. The operator $C$ is determined dynamically and in most cases perturbatively. This approach succeeded in correcting the negative norm and unitarity problems.

The other approach, due to Mostafazadeh [19], has generalized the requirement of a non-Hermitian theory to have a real spectrum to the existence of a positive definite metric operator $\eta$ such that $H^{\dagger}=\eta H \eta^{-1}$. The existence of the metric operator assures the existence of a similarity transformation which has the job of transforming the non-Hermitian Hamiltonian operator $H$ into a Hermitian operator $h$, where $h=\rho^{-1} H \rho$ and $\rho=\sqrt{\eta}$.

In both approaches described above, the metric operator $\eta$ and the $C$ operator are assumed to be composed of the dynamical operators (momentum and position operators) in the Hamiltonian. While these approaches can be applied in both the quantum mechanical and quantum field versions of a Hamiltonian, their application in field theory leads to complicated $\eta$ and $C$ operators [20]. This is the reason why in the literature one can find even up to seventh order calculation of $C$ for the $i x^{3}$ while only a first order at low space-time dimensions calculation of the same operator exists for the quantum field $i \phi^{3}$ scalar theory. In this work, we introduce a new ansatz that makes the calculation of the metric operator in field theory goes simple as in the quantum mechanical case. The idea is that, instead of conjecturing the shape of the metric operator as a functional of the field $\phi$ and its conjugate momentum field $\pi$, we assume that it can also include the field gradient $\nabla \phi$. As we will see this assumption will simplify the calculation and lead to metric operator with parameters that do not depend on the dimension of space-time. Note that the metric operator is not unique and one can find more than one metric operator for the same theory.

To start, consider the Hamiltonian model of the form;

$$
H=\frac{1}{2}\left((\nabla \phi)^{2}+\pi^{2}+m^{2} \phi^{2}\right)+i g \phi^{3},
$$


where $\phi$ is the field operator, $\pi$ is the conjugate momentum and $g$ is the coupling constant. This theory is non-Hermitian but $\mathcal{P} \mathcal{T}$-symmetric and thus is physically acceptable as it has a real spectrum. Also, following Mostafazadeh, the metric operator can be assumed to have the form $\eta=\exp (-Q)$, where $Q$ is Hermitian. Note that, although the Hamiltonian in Eq.(11) is non-Hermitian in a Hilbert space with the Dirac sense inner product, it is Hermitian in a Hilbert space endowed by the inner product $\langle n|\eta| n\rangle$.

To obtain $Q$, we rename the terms in the Hamiltonian as;

$$
\begin{gathered}
H=H_{0}+g H_{I} \\
H_{0}=\frac{1}{2} \int d^{3} x\left((\nabla \phi)^{2}+\pi^{2}+m^{2} \phi^{2}\right), \\
H_{I}=i \int d^{3} x \phi^{3},
\end{gathered}
$$

and in using the relation $H^{\dagger}=\eta H \eta^{-1}$, we get

$$
\begin{aligned}
H^{\dagger} & =\exp (-Q) H \exp (Q)=H+[-Q, H]+\frac{[-Q,[-Q, H]]}{2 !} \\
& +\frac{[-Q,[-Q,[-Q, H]]]}{3 !}+\ldots .
\end{aligned}
$$

where

$$
Q=Q_{0}+g Q_{1}+g^{2} Q_{2}++g^{3} Q_{3}+. .
$$

Accordingly;

$$
\begin{aligned}
& -2 g H_{I}=\left[-\left(Q_{0}+g Q_{1}+g^{2} Q_{2}++g^{3} Q_{3}+\ldots\right), H\right] \\
& {\left[-\left(Q_{0}+g Q_{1}+g^{2} Q_{2}++g^{3} Q_{3}+\ldots\right),\right.} \\
& +\frac{\left.\left[-\left(Q_{0}+g Q_{1}+g^{2} Q_{2}++g^{3} Q_{3}+\ldots\right), H\right]\right]}{2 !} \\
& +\frac{\left[\begin{array}{c}
{\left[-\left(Q_{0}+g Q_{1}+g^{2} Q_{2}++g^{3} Q_{3}+\ldots\right),\right.} \\
{\left[-\left(Q_{0}+g Q_{1}+g^{2} Q_{2}++g^{3} Q_{3}+\ldots\right),\right.} \\
\left.\left[-\left(Q_{0}+g Q_{1}+g^{2} Q_{2}++g^{3} Q_{3}+\ldots\right), H\right]\right]
\end{array}\right]}{3 !} \\
& +\ldots \ldots \ldots \ldots \ldots \ldots \ldots \ldots \ldots \ldots
\end{aligned}
$$


Then by equating coefficients of $g^{n}$ in each side, we get the first seven orders as follows [27];

$$
\begin{aligned}
& g^{1}:-2 H_{I}=\left[-Q_{1}, H_{0}\right] \\
& g^{3}: 0=\left[-Q_{3}, H_{0}\right]+\frac{\left[-Q_{1},\left[-Q_{1},\left[-Q_{1}, H_{0}\right]\right]\right]}{3 !}+\frac{\left[-Q_{1},\left[-Q_{1}, H_{I}\right]\right]}{2 !} \\
& g^{5}: 0=\left[-Q_{5}, H_{0}\right]+\frac{\left[-Q_{1},\left[-Q_{1},\left[-Q_{1},\left[-Q_{1},\left[-Q_{1}, H_{0}\right]\right]\right]\right]\right]}{5 !}+\frac{\left[-Q_{3},\left[-Q_{1},\left[-Q_{1}, H_{0}\right]\right]\right]}{3 !} \\
& +\frac{\left[-Q_{1},\left[-Q_{3},\left[-Q_{1}, H_{0}\right]\right]\right]}{3 !}+\frac{\left[-Q_{1},\left[-Q_{1},\left[-Q_{3}, H_{0}\right]\right]\right]}{3 !} \\
& +\frac{\left[-Q_{1},\left[-Q_{1},\left[-Q_{1},\left[-Q_{1}, H_{I}\right]\right]\right]\right]}{4 !}+\frac{\left[-Q_{1},\left[-Q_{3}, H_{I}\right]\right]}{2 !}+\frac{\left[-Q_{3},\left[-Q_{1}, H_{I}\right]\right]}{2 !}, \\
& g^{7}: 0=\left[-Q_{7}, H_{0}\right]+\frac{\left[-Q_{1},\left[-Q_{1},\left[-Q_{1},\left[-Q_{1},\left[-Q_{1},\left[-Q_{1},\left[-Q_{1}, H_{0}\right]\right]\right]\right]\right]\right]\right]}{7 !} \\
& +\frac{\left[-Q_{1},\left[-Q_{1},\left[-Q_{1},\left[-Q_{1},\left[-Q_{3}, H_{0}\right]\right]\right]\right]\right]}{5 !} \\
& +\frac{\left[-Q_{1},\left[-Q_{1},\left[-Q_{1},\left[-Q_{3},\left[-Q_{1}, H_{0}\right]\right]\right]\right]\right]}{5 !}+\frac{\left[-Q_{1},\left[-Q_{1},\left[-Q_{3},\left[-Q_{1},\left[-Q_{1}, H_{0}\right]\right]\right]\right]\right]}{5 !} \\
& +\frac{\left[-Q_{1},\left[-Q_{3},\left[-Q_{1},\left[-Q_{1},\left[-Q_{1}, H_{0}\right]\right]\right]\right]\right]}{5 !}+\frac{\left[-Q_{3},\left[-Q_{1},\left[-Q_{1},\left[-Q_{1},\left[-Q_{1}, H_{0}\right]\right]\right]\right]\right]}{5 !} \\
& +\frac{\left[-Q_{3},\left[-Q_{3},\left[-Q_{1}, H_{0}\right]\right]\right]}{3 !}+\frac{\left[-Q_{3},\left[-Q_{1},\left[-Q_{3}, H_{0}\right]\right]\right]}{3 !}+\frac{\left[-Q_{1},\left[-Q_{3},\left[-Q_{3}, H_{0}\right]\right]\right]}{3 !} \\
& +\frac{\left[-Q_{1},\left[-Q_{1},\left[-Q_{5}, H_{0}\right]\right]\right]}{3 !}+\frac{\left[-Q_{1},\left[-Q_{5},\left[-Q_{1}, H_{0}\right]\right]\right]}{3 !}+\frac{\left[-Q_{5},\left[-Q_{1},\left[-Q_{5}, H_{0}\right]\right]\right]}{3 !} \\
& +\frac{\left[-Q_{1},\left[-Q_{1},\left[-Q_{1},\left[-Q_{1},\left[-Q_{1},\left[-Q_{1}, H_{I}\right]\right]\right]\right]\right]\right]}{6 !}+\frac{\left[-Q_{3},\left[-Q_{3}, H_{I}\right]\right]}{2 !} \\
& +\frac{\left[-Q_{1},\left[-Q_{1},\left[-Q_{1},\left[-Q_{3}, H_{I}\right]\right]\right]\right]}{4 !}+\frac{\left[-Q_{1},\left[-Q_{1},\left[-Q_{3},\left[-Q_{1}, H_{I}\right]\right]\right]\right]}{4 !} \\
& +\frac{\left[-Q_{1},\left[-Q_{3},\left[-Q_{1},\left[-Q_{1}, H_{I}\right]\right]\right]\right]}{4 !}+\frac{\left[-Q 3,\left[-Q_{1},\left[-Q_{1},\left[-Q_{1}, H_{I}\right]\right]\right]\right]}{4 !}+\frac{\left[-Q_{5},\left[-Q_{1}, H_{I}\right]\right]}{2 !} \\
& +\frac{\left[-Q_{1},\left[-Q_{5}, H_{I}\right]\right]}{2 !} \text {. }
\end{aligned}
$$

In the literature, the $Q$ operator is assumed to depend only on the position and momentum operators [20]. This assumption has been mapped to quantum field problems with the $Q$ operator is a functional in the field $\phi$ and its canonical conjugate field $\pi$. However, there exists an operator in the Hamiltonian $(\nabla \phi)$ which has no analog in the quantum mechanical case. Accordingly, it makes sense to extend the assumption for the $Q$ operator to be a functional of $\phi, \pi$ and $\nabla \phi$ fields. Accordingly, one can conjecture the form of $Q_{1}$ to be;

$$
\begin{aligned}
Q_{1} & =C_{1} \int d^{3} z \pi^{3}(z)+\frac{C_{2}}{3} \int d^{3} z\left(\pi(z) \phi^{2}(z)+\phi(z) \pi(z) \phi(z)+\phi^{2}(z) \pi(z)\right) \\
& +\frac{C_{3}}{3} \int d^{3} z(\pi(z) \nabla \phi(z) \nabla \phi(z)+\nabla \phi(z) \pi(z) \nabla \phi(z)+\nabla \phi(z) \nabla \phi(z) \pi(z)) .
\end{aligned}
$$


To find out the parameters $C_{1}, C_{2}$ and $C_{3}$ we consider the first equation in the above set;

$$
-2 H_{I}=\left[-Q_{1}, H_{0}\right]
$$

Let us commute each term in $Q_{1}$ with each term in $H_{0}$.

First, consider the commutator of the first term in $Q_{1}$ with each term in $H_{0}$;

$$
\begin{aligned}
& C_{1} \int d^{3} x \int d^{3} z\left[\pi^{3}(z), \frac{1}{2} \nabla_{x} \phi(x) \nabla_{x} \phi(x)\right], \\
& =\frac{1}{2} C_{1} \int d^{3} x \int d^{3} z\left[\pi^{3}(z), \nabla_{x} \phi(x)\right] \nabla_{x} \phi(x) \\
& +\frac{1}{2} C_{1} \int d^{3} x \int d^{3} z \nabla_{x} \phi(x)\left[\pi^{3}(z), \nabla_{x} \phi(x)\right], \\
& =\frac{1}{2} C_{1} \int d^{3} x \int d^{3} z \nabla_{x}\left[\pi^{3}(z), \phi(x)\right] \nabla_{x} \phi(x) \\
& +\frac{1}{2} C_{1} \int d^{3} x \int d^{3} z \nabla_{x} \phi(x) \nabla_{x}\left[\pi^{3}(z), \phi(x)\right], \\
& =\frac{3}{2} C_{1} \int d^{3} x \int d^{3} z \pi^{2}(z) \nabla_{x}\left(-i \delta^{3}(z-x) \nabla_{x} \phi(x),\right. \\
& +\frac{3}{2} C_{1} \int d^{3} x \int d^{3} z \nabla_{x} \phi(x) \pi^{2}(z) \nabla_{x}\left(-i \delta^{3}(z-x),\right. \\
& =\frac{3}{2} C_{1} \int d^{3} x \int d^{3} z \pi^{2}(z) \nabla_{x}\left(i \delta^{3}(x-z) \nabla_{x} \phi(x),\right. \\
& +\frac{3}{2} C_{1} \int d^{3} x \int d^{3} z \nabla_{x} \phi(x) \pi^{2}(z) \nabla_{x}\left(i \delta^{3}(x-z),\right. \\
& =-\frac{3 i}{2} C_{1} \int d^{3} x \pi^{2}(x) \nabla_{x}^{2} \phi(x)-\frac{3 i}{2} C_{1} \int d^{3} x \nabla_{x}^{2} \phi(x) \pi^{2}(x) .
\end{aligned}
$$

Also

$$
\begin{aligned}
& \frac{1}{2} m^{2} C_{1} \int d^{3} x \int d^{3} z\left[\pi^{3}(z), \phi(x) \phi(x)\right] \\
& =\frac{1}{2} m^{2} C_{1} \int d^{3} x \int d^{3} z\left[\pi^{3}(z), \phi(x)\right] \phi(x) \\
& +\frac{1}{2} m^{2} C_{1} \int d^{3} x \int d^{3} z \phi(x)\left[\pi^{3}(z), \phi(x)\right] \\
& =\frac{3}{2} m^{2} C_{1} \int d^{3} x \int d^{3} z \pi^{2}(z)\left(-i \delta^{3}(x-z) \phi(x)\right. \\
& +\frac{3}{2} m^{2} C_{1} \int d^{3} x \int d^{3} z \phi(x) \pi^{2}(z)\left(-i \delta^{3}(x-z)\right. \\
& =\frac{-3 i}{2} m^{2} C_{1} \int d^{3} x\left(\pi^{2}(x) \phi(x)+\phi(x) \pi^{2}(x)\right)
\end{aligned}
$$

For the second term in $Q_{1}$ with each term in $H_{0}$, we get 


$$
\begin{aligned}
& \frac{C_{2}}{6} \int d^{3} x \int d^{3} z\left[\pi(z) \phi^{2}(z)+\phi(z) \pi(z) \phi(z)+\phi^{2}(z) \pi(z), \pi^{2}(x)\right] \\
& =i C_{2} \int d^{3} x\left(\phi(x) \pi^{2}(x)+\pi^{2}(x) \phi(x)\right)
\end{aligned}
$$

Now

$$
\begin{aligned}
& \frac{C_{2}}{6} \int d^{3} x \int d^{3} z\left[\pi(z) \phi^{2}(z), \nabla_{x} \phi(x) \nabla_{x} \phi(x)\right] \\
& =\frac{C_{2}}{6} \int d^{3} x \int d^{3} z \nabla_{x}\left(-i \delta^{3}(z-x)\right) \nabla_{x} \phi(x) \phi^{2}(z) \\
& +\frac{C_{2}}{6} \int d^{3} x \int d^{3} z \nabla_{x} \phi \nabla_{x}\left(-i \delta^{3}(z-x)\right)(x) \phi^{2}(z) \\
& =-\frac{i C_{2}}{3} \int d^{3} x \nabla_{x}^{2} \phi(x) \phi^{2}(x) .
\end{aligned}
$$

Then

$$
\begin{aligned}
& \frac{C_{2}}{6} \int d^{3} x \int d^{3} z\left[\pi(z) \phi^{2}(z)+\phi(z) \pi(z) \phi(z)+\phi^{2}(z) \pi(z), \nabla_{x} \phi(x) \nabla_{x} \phi(x)\right] \\
& =-i C_{2} \int d^{3} x \nabla_{x}^{2} \phi(x) \phi^{2}(x),
\end{aligned}
$$

and

$$
\begin{aligned}
& \frac{C_{2} m^{2}}{6} \int d^{3} x \int d^{3} z\left[\pi(z) \phi^{2}(z)+\phi(z) \pi(z) \phi(z)+\phi^{2}(z) \pi(z), \phi(x) \phi(x)\right], \\
& =-i C_{2} m^{2} \int d^{3} x \phi^{3}(x) .
\end{aligned}
$$

For the third term of $Q_{1}$ with each term in $H_{0}$;

$$
\begin{aligned}
& \frac{C_{3}}{6} \int d^{3} x \int d^{3} z\left[\nabla_{z} \phi(z) \nabla_{z} \phi(z) \pi(z)+\nabla_{z} \phi(z) \pi(z) \nabla_{z} \phi(z)+\pi(z) \nabla_{z} \phi(z) \nabla_{z} \phi(z), \pi^{2}(x)\right], \\
& =\frac{i C_{3}}{2} \int d^{3} x \pi(x) \pi(x) \nabla^{2} \phi(x)+\frac{i C_{3}}{2} \int d^{3} x \nabla^{2} \phi(x) \pi(x) \pi(x) .
\end{aligned}
$$

Now consider;

$$
\begin{aligned}
\frac{C_{3}}{6} \int d^{3} x \int d^{3} z\left[\pi(z) \nabla \phi(z) \nabla \phi(z), \nabla_{x} \phi(x) \nabla_{x} \phi(x)\right] & =\frac{2 i C_{3}}{6} \int d^{3} x \nabla \frac{(\nabla \phi(x))^{3}}{3} \\
& =\frac{2 i C_{3}}{6} \int d \frac{(\nabla \phi(x))^{3}}{3}
\end{aligned}
$$

which is an integration of a total derivative that can be integrated out and thus vanish. 
Also,

$$
\begin{aligned}
& \frac{m^{2} C_{3}}{6} \int d^{3} x \int d^{3} z\left[\begin{array}{c}
\nabla_{z} \phi(z) \nabla_{z} \phi(z) \pi(z)+\nabla_{z} \phi(z) \pi(z) \nabla_{z} \phi(z) \\
+\pi(z) \nabla_{z} \phi(z) \nabla_{z} \phi(z), \phi(x) \phi(x)
\end{array}\right], \\
& =\frac{i m^{2} C_{3}}{2} \int d^{3} x \phi^{2}(x) \nabla^{2} \phi(x) .
\end{aligned}
$$

Note that, the calculations above used integration by parts as well as employed the different properties of the derivative of the Dirac delta function.

Now for the relation $\left[Q_{1}, H_{0}\right]=2 H_{I}$ to be verified, one have to take

$$
C_{2}=\frac{-2}{m^{2}}, \quad C_{1}=-\frac{4}{3} \frac{1}{m^{4}}, C_{3}=\frac{-4}{m^{4}} .
$$

To realize how advantageous is our ansatz used in this work, compare the metric operator obtained here with that obtained in Ref. [20]. To shed light on the differences between the two results we note that although the algorithm used in Ref.[20] can applied to the field theory in Eq.(11) for the calculation of the $Q$ operator, the results their have parameters which depend on the space-time dimensions while our result is general and can be used for any space-time dimensions. Moreover, while the from in Ref.[20] is complicated and nonlocal, the form we obtained is local and simple and thus lead to simpler calculations of the Physical amplitudes. Besides, the modification we add here to the form of the $Q$ operator makes the higher orders calculations in quantum field problems as simple as those in the quantum mechanical case and can, in fact, be conjectured from the corresponding results in the literature for the $i x^{3}$ model. However, the higher order calculations as well as detailed analysis of the theory under consideration is postponed to appear in another article.

To conclude, we have introduced a new ansatz for the metric operator calculation in quantum field theory. Without the ansatz introduced in this work, the metric operator in field theory is cumbersome and non-local.

We applied the ansatz for the $i \phi^{3}$ scalar field theory for which we obtained the metric operator up to first order in the coupling constant $g$. We realized that the parameters appearing in the form of the metric operator are just the original parameters in the Hamiltonian model which are independent on the dimension of the space-time. This result is very important as for the current algorithms in the literature one has to do the calculations for a fixed space-time dimensions. Moreover, the previous calculations of the metric operator resulted in a very complicated form which in turn will make the calculations of the physical 
amplitudes very. On the other hand our ansatz results in a shape that is very similar to the quantum mechanical shape of the metric operator and though we have done the calculations up to first order, one can conjecture the higher orders from the available metric operator in $0+1$ dimensions (quantum mechanics). The importance of this work stems from the fact that the effective field approach of the more important $-\phi^{4}$ has been proved to be suc-

cessful in studying this theory [1]. In fact, the effective from of the $-\phi^{4}$ is real-line theory with an $i \phi^{3}$ non-Hermitian term and thus with the aid of this work a serious study of the $\mathcal{P} \mathcal{T}$-symmetric Higgs mechanism is now available.

\section{Acknowledgments}

The author would like to thank Dr. S. A. Elwakil for his and kind help. Deep thanks to the Physics department in Qassim university for support and help. 
[1] Abouzeid shalaby,Phys. Rev. D. 79, 065017 (2009)

[2] C. M. Bender, S. Boettcher, and P. N. Meisinger, J. Math. Phys. 40, 2201 (1999).

[3] Carl M Bender, Daniel W Hook and Lawrence R Mead, J.Phys.A41:392005 (2008 )

[4] Carl M. Bender, Peter N. Meisinger, and Haitang Yang, Phys. Rev. D63, 045001 (2001).

[5] C. M. Bender and S. Boettcher, Phys. Rev. Lett. 80, 5243 (1998).

[6] C. M. Bender, F. Cooper, P. N. Meisinger, and V. M. Savage, Phys. Lett. A 259, 224 (1999).

[7] C. M. Bender and G. V. Dunne, J. Math. Phys. 40, 4616 (1999).

[8] E. Delabaere and F. Pham, Phys. Lett. A 250, 25 (1998) and 29 (1998).

[9] E. Delabaere and D. T. Trinh, J. Phys. A: Math. Gen. 33, 8771 (2000).

[10] G. A. Mezincescu, J. Phys. A: Math. Gen. 33, 4911 (2000).

[11] C. M. Bender, S. Boettcher, and V. M. Savage, J. Math. Phys. 41, 6381 (2000).

[12] C. M. Bender and Q. Wang, J.Phys. A34, 9835 (2001).

[13] K. C. Shin, University of Illinois preprint.

[14] C. M. Bender and E. J. Weniger, J. Math. Phys. 42, 2167 (2001).

[15] C. M. Bender, G. V. Dunne, P. N. Meisinger, and M.Simsek, Phys. Lett. A 281, 311 (2001).

[16] P. Dorey, C. Dunning, and R. Tateo, hep-th/0103051.

[17] C. M. Bender, M. Berry, P. N. Meisinger, V. M. Savage, and M. S.ims. ek, J. Phys. A:Math. Gen. 34, L31-L36 (2001).

[18] Carl M. Bender, Jun-Hua Chen, Kimball A. Milton, J.Phys.A39, 1657 (2006).

[19] A. Mostafazadeh, J. Phys. A: Math. Gen. 38, 6557 (2005) and Erratum 38, 8185 (2005).

[20] Carl M. Bender, Dorje C. Brody and Hugh F. Jones, Phys.Rev. D70 (2004) 025001; Erratumibid. D71 (2005) 049901.

[21] Carl M. Bender, Sebastian F. Brandt, Jun-Hua Chen, Qing-hai Wang, Phys.Rev.D71, 025014 (2005).

[22] Carl M. Bender, Dorje C. Brody, and Hugh F. Jones, Phys.Rev.D70:025001 (2004), Erratumibid.D71:049901 (2005).

[23] Carl. M. Bender, International Journal of Modern Physics A20, No.19 2646 (2005).

[24] Abouzeid shalaby, Eur.Phys.J.C50:999-1006 (2007).

[25] A. Mostafazadeh, J. Math. Phys., 43, 3944 (2002). 
[26] A. Mostafazadeh, J. Math. Phys. 43, 205 (2002).

[27] This set of equations have been obtained in Ref.[20] but they employed the results from each equation in the next one. 